\title{
Latest Advances in Nanoscale Chemical Imaging and Spectroscopy
}

Anirban Roy

\author{
Bruker Nano Surfaces, Goleta, California, United States
}

For the last few decades the rapid growth in the field of nanoscience and technology has led to the development of new characterization tools for nanoscale materials. Traditional IR and Raman spectroscopy and imaging offers excellent chemical insights; however, the spatial resolution is limited by the optical diffraction limit $(\sim 1 / 2)$. Although, recent Super-resolution microscopy techniques $[1,2]$ offer superior spatial resolution, they are primarily implemented in fluorescence imaging, hence needs external fluorophore tag for detection. Alternatively, nanoscale IR spectroscopy/imaging offers a "tag free" spectral detection with high spatial resolution beyond optical diffraction limit (2-5 $\mathrm{mm}$ ) by exploiting an AFM probe [3] to detect either photothermal expansion force (PTIR/AFM-IR) or near field scattered IR light (s-SNOM).

Recent developments in PTIR/AFM-IR and s-SNOM technology have significantly augmented the speed and spatial resolution for chemical analysis. One of the new developments (Tapping AFM-IR) allows acquisition of IR images at a specific absorption band simultaneously with sample topography and viscoelastic properties, providing a complete set of topographical, chemical and mechanical insights with $\sim 10 \mathrm{~nm}$ spatial resolution. These high-resolution measurements are currently accompanied by high speed tunable lasers with broad spectral coverage ranging from $800-3600 \mathrm{~cm}^{-1}$, encompassing full capability of traditional FTIR characterization for dimensions orders of magnitudes smaller than the optical diffraction limit. For example, Figure 1c shows topography and chemical map of PS- $b$-LDPE block copolymer on a silicon substrate. IR images at $1466 / 2920 \mathrm{~cm}^{-1}$ and $1488 / 3032 \mathrm{~cm}^{-1}$ highlight the distribution of lowdensity polyethylene (LDPE) and polystyrene (PS), respectively with feature sizes $\sim 50 \mathrm{~nm}$ or smaller. Along with high spatial resolution, high speed spectral acquisition enables acquiring hyperspectral data cube for rigorous statistical analysis relevant to Chemometrics applications.

In this presentation, we will highlight the technical background and applications of these emerging technologies in different fields, e.g., nanomaterials, life sciences, polymers, microelectronics etc. 

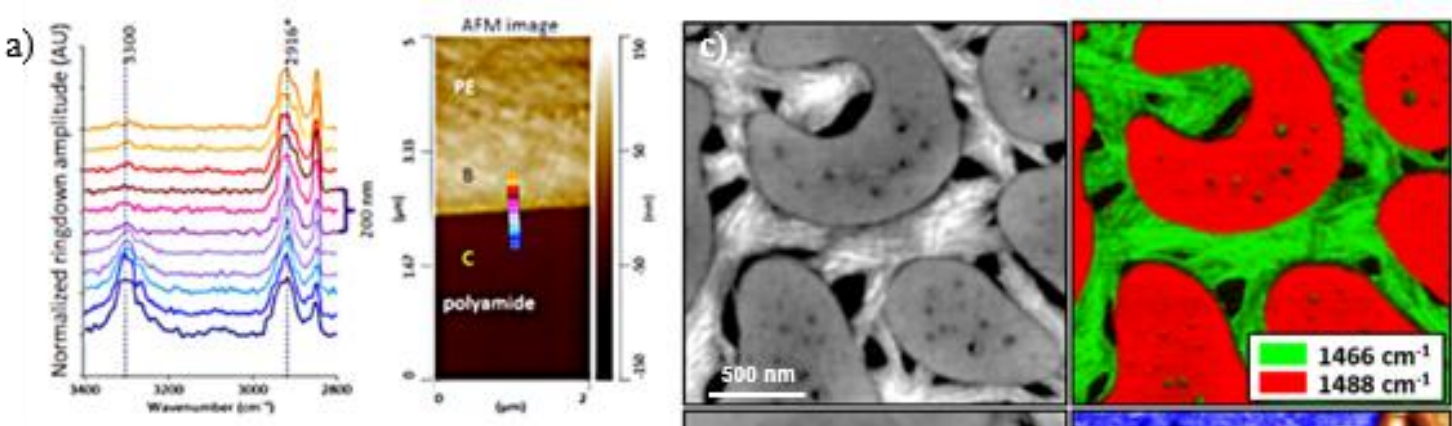

b)
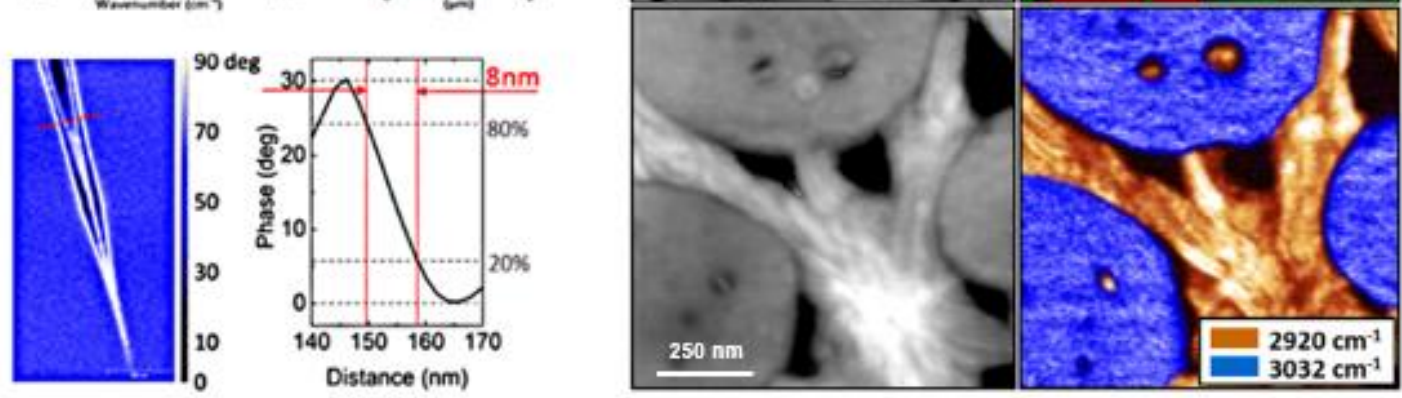

Figure 1. Photothermal IR/AFM-IR measurements at the polyethylene (PE) | Polyamide (PA) interface illustrating change in IR spectral features at the phase boundary (a). s-SNOM image of a graphene wedge on silicon substrate showing standing waves of the surface plasmon polaritons on graphene surface with $\sim 8 \mathrm{~nm}$ spatial resolution (b). Topography (grayscale) and Tapping AFM-IR images of PS-b-LDPE copolymers at different IR absorption bands highlighting the chemical composition (green/orange: LDPE | red/blue: PS).

\section{References}

[1] B Huang et al., Annu. Rev. Biochem., 78 (2009), p. 993-1016

[2] T D Harris et al, Appl. Spectrosc., 48 (1994), p. 14A-21A

[3] A Dazzi and C B Prater, Chem. Rev., 117 (2016), p. 5146-5173 